Neil Louis L. Apale, MD Joel A. Romualdez, MD Rodolfo E. Rivera, MD

Department of Otolaryngology Head and Neck Surgery St. Luke's Medical Center

\section{Rosai-Dorfman Disease Presenting As Recurrent Nasal Polyposis}

\begin{abstract}
Objectives: To present a case of Rosai-Dorfman disease in an individual with a 14-year history of recurrent nasal polyposis and discuss its clinical presentation, physical examination, radiologic findings, histopathologic characteristics and available treatment.
\end{abstract}

\section{Methods:}

\author{
Design: Case Report \\ Setting: Tertiary Government Hospital \\ Patient: One
}

Results: A 26-year-old Filipino diagnosed and repeatedly treated medically and surgically for recurrent nasal polyposis underwent repeat endoscopic sinus surgery. A histopathologic impression of Rosai-Dorfman disease was confirmed by positive S-100 and CD1a negative immunochemistry in conjunction with the morphologic findings.

Conclusion: Rosai-Dorfman disease is a rare entity which should be considered when dealing with recurrent nasal polyposis that is intractable to initial medical and surgical therapies. Histopathologic findings of emperipolesis and immunohistochemical S-100 stains play a key role in the diagnosis but there is yet no definite treatment for this disease.

\section{Keywords: recurrent nasal polyposis, Rosai-Dorfman disease, emperipolesis, S-100 stain}

Nasal polyps are common pathology of unknown etiology with a high rate of recurrence after surgery. ${ }^{1}$ A study of 148 patients to determine the rate and reasons for polyp recurrence after functional endoscopic sinus surgery showed recurrence in 74 patients (49.3\%) in the first two years after surgery and 17 patients (12.2\%) in the next 3-4 years. There were no associations noted between allergy, infection and recurrence of polyps.' Another study that analyzed 10 variables (age, gender, history of purulent nasal discharge, facial pain, anosmia, post nasal drip, headache, nasal allergy, asthma and CT scan staging using the Lund-McKay scoring system) for association with polyp recurrence found a 19\% recurrence rate and no association with the variables analyzed except for $\mathrm{CT}$ staging which was significantly higher among the group with recurrence compared to the group without recurrence $(p<0.00){ }^{2}$

Recurrent polyposis which is unresponsive to the standard therapy is associated with hyperplastic chronic sinusitis, allergy, cystic fibrosis and acetylsalicylic (ASA) sensitivity. Patients with ASA sensitivity typically have adult onset asthma with nasal polyps and chronic sinusitis. ${ }^{2}$ Another disease entity associated with recurrent nasal polyposis is inverting papilloma which is a benign but locally aggressive tumor of the nasal cavity. It is similar in appearance but is fleshier than nasal polyp. Histopathology can distinguish the one from the other. ${ }^{2}$

We present a case of recurrent nasal polyposis not responding to repeated medical and surgical treatments diagnosed with Rosai-Dorfman disease. 


\section{CASE REPORTS}

\section{CASE REPORT}

A 26-year-old man with recurrent nasal obstruction and nasal discharge for the past 14 years underwent 12 functional endoscopic sinus surgeries in 12 years for chronic rhinosinusitis with nasal polyposis. Although his past medical and social histories were unremarkable, he had a family history of bronchial asthma (mother), hypertension (father), and skin malignancy (aunt).

He presented to us due to recurrence of nasal symptoms. Physical examination showed nasal polyps appearing as non-translucent, coarse and pinkish polypoid masses. Paranasal sinus $\mathrm{CT}$ scans revealed erosive changes in the posterior wall of the right maxillary antrum with bone thinning and erosive changes in the ethmoids with nodular densities in the nasal cavity. (Figure 1) Flexible nasal endoscopy showed the extent of the nasal polyps. (Figure 2) Endoscopic sinus surgery was performed and histopathologic evaluation of the extracted nasal polyps revealed "eosinophilic granularity within the histiocytes and occasional lymphocytes present within the histiocytic cytoplasm with no malignant neoplastic tissue detected." (Figure3) With an impression of Rosai-Dorfman disease, immunohistochemical stains for confirmation were positive for $\mathrm{S} 100$ and negative for $\mathrm{CD1}$, which, coupled with the morphology favored the diagnosis. Post-operative endoscopicallyguided nasal douches of isotonic nasal saline powder (Flo) sachet, 1 sachet $/ 200 \mathrm{~mL}$ distilled water and budesonide (Pulmicort) $1 \mathrm{mg} / 2 \mathrm{~mL}$ per nebule, 1 nebule/ $200 \mathrm{~mL}$ distilled water were started every other day during the first post-operative month and gradually reduced in frequency with resolution of the black crusting admixed with whitish to yellowish mucoid secretions in the nasal cavity. At present, the patient is symptom-free on regular nasal douching twice a week. (Figure 4)

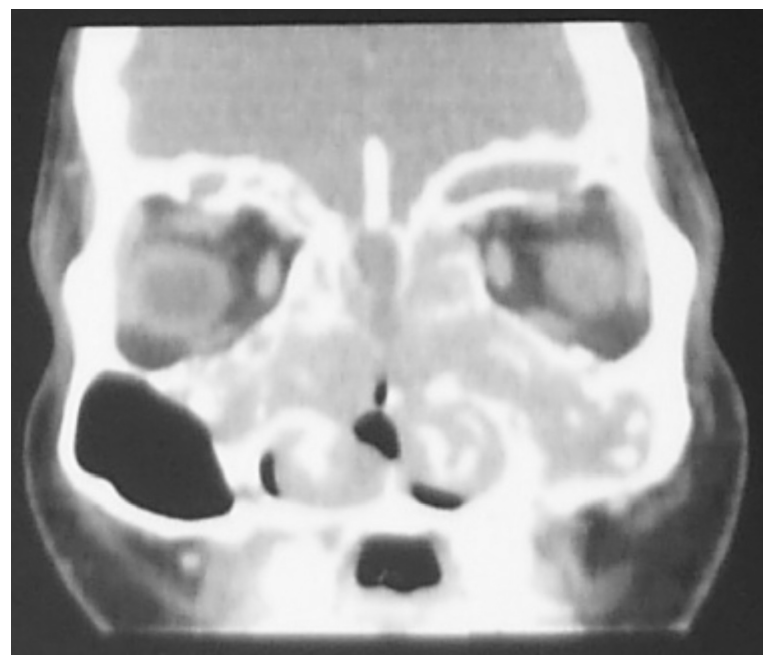

Figure 1. Coronal contrast CT scan showing complete opacification of the left maxillary and bilateral ethmoid sinuses. The nasal septum is moderately deviated to the right

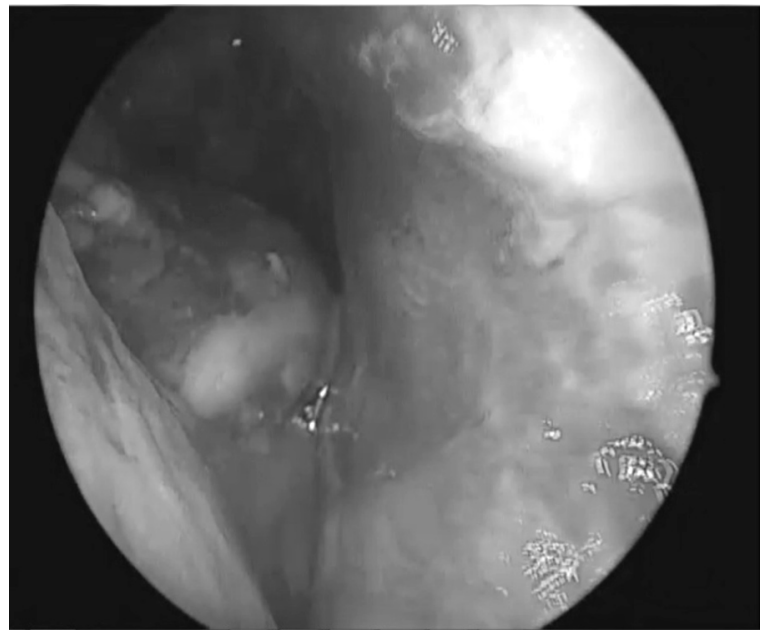

Figure 2. Translucent coarse pinkish lesion in the nasal cavity seen on flexible nasal endoscopy

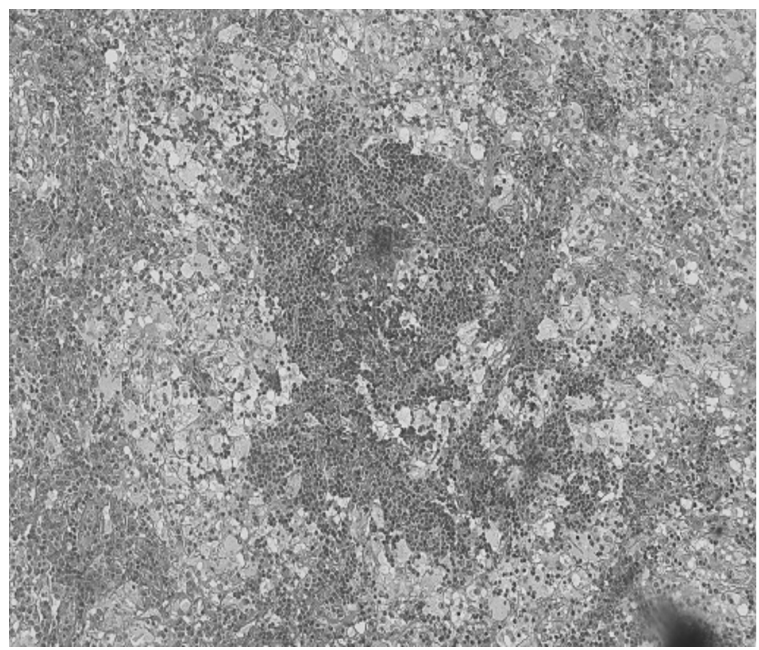

Figure 3. Microscopic view showing engulfment of lymphocytes by histiocytes (emperipolesis)

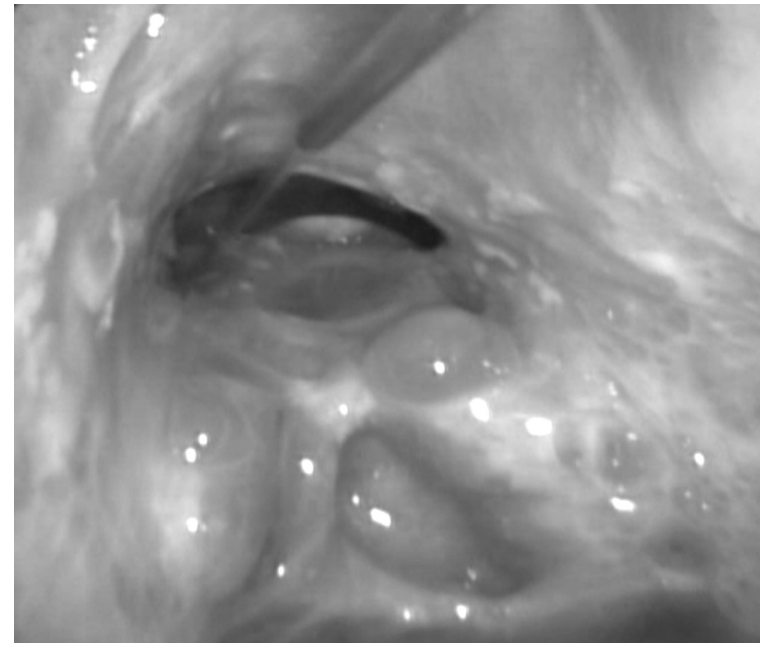

Figure 4. Frontal Sinus with polypoid mucosa and good mucosal healing 6 weeks post-surgery and regular nasal douching 


\section{DISCUSSION}

Rosai-Dorfman Disease (RDD) is a rare non-neoplastic lymphoproliferative histiocytic disorder. ${ }^{3}$ It is more frequently seen in children and young adults but may occur in any age group and is more common in males and those of African descent. ${ }^{4}$ The classical clinical presentation of RDD is sinus histiocytosis with massive and painless cervical lymphadenopathy. ${ }^{5}$ However, extranodal disease occurs in up to $43 \%$ of patients and among these, $75 \%$ of cases occur in the head and neck region. Involvement of the nasal cavity presents with recurrent nasal obstruction and discomfort and on examination reveals small red-wine colored tumor masses. ${ }^{6}$ CT scan findings in Rosai-Dorfman Disease with involvement of the paranasal sinuses shows diffuse mucoperiosteal thickening of the sinuses with nodular densities in the nasal cavity which are similar findings found in Chronic Rhinosinusitis with Nasal polyposis. ${ }^{5}$ Imaging by CT scan demonstrates the extent of the disease but histopathology and immunostaining are needed for definite diagnosis. Histological features of the disease include marked proliferation of sinus histiocytes which often contain phagocytosed lymphocytes (emperipolesis). ${ }^{6}$ The presence of emperipolesis or the engulfment of lymphocytes and erythrocytes by histiocytes that express S-100 is considered diagnostic of Rosai-Dorfman Disease.
At present, there is still no definite treatment for this disease. Systemic corticosteroids are usually helpful in decreasing nodal size and symptoms, however they can be quite immunosuppressive and recurrence of lesions can occur after a short period of interruption. Surgical management may be required if there are obstructive or compressive symptoms as in our patient with nasal obstruction.?

In this case, Rosai-Dorfman Disease presented as recurrent nasal polyposis. Surgical removal of the lesions is the primary treatment and nasal douching is advocated as an adjunct therapy for recurrent nasal polyposis. Saline nasal irrigation demonstrates improvement in symptoms and quality of life. It promotes mucociliary clearance by flushing out mucus, crusts and irritants. Adding Budesonide respules to nasal douching has been observed to decrease mucosal inflammation, shortening the stage of epithelialisation and accelerating recovery of mucosa after FESS. ${ }^{8}$

Rosai-Dorfman disease is a rare entity which should be considered when dealing with recurrent nasal polyposis that is intractable to initial medical and surgical therapies. Histopathologic findings of emperipolesis and immunohistochemical S-100 stains play a key role in the diagnosis, but there is yet no definite treatment for this disease.

\footnotetext{
REFERENCES

1. Eitan Y, Jacob S, Tamara D, Rami T, Tuvia H. Recurrence of Nasal Polyps After Functional Endoscopic Sinus Surgery. Conexiuni Medicale 2009 Dec; 16: 27-9.

2. Akhtar S, Ikram M, Azam I, Dahri T. Factors associated with recurrent nasal polyps: a tertiary care experience. J Pak Med Assoc, 2010 Feb; 60(2) : 102-4.

3. Rosai J, Dorfman RF. Sinus histiocytosis with massive lymphadenopathy: A newly recognized benign clinicopathological entity. Arch Pathol 1969 Jan; 87(1) : 63-70.

4. Komp DM. The treatment of sinus histiocytosis with massive lymphadenopathy (Rosai Dorfman disease). Semin Diagn Pathol 1990 Feb; 7(1): 83-6.

5. La Barge DV, Salzman KL, Harnsberger HR, Ginsberg LE, Hamilton BE, Wiggins RH, Hudgins PA. Sinus Histiocytosis with Massive Lymphadenopathy (Rosai-Dorfman Disease): Imaging Manifestations in the Head and Neck. AJR 2008 Dec; 191(6):W299-W306. 10.2214/AJR.08.1114

6. Hagemann M, Zbaren P, Stauffer E, Caversaccio M. Nasal and Paranasal Sinus Manifestation of Rosai-Dorfman Disease. Rhinology 2005 Sep; 43(3):229-32.

7. Huang YT, Ng SH, Ko SF, Wong HF, Chen YL, Huang MC, Toh CH, Wai YY. Extranodal RosaiDorfman Disease with Paranasal Sinuses and Intracranial Involvement: A Case Report. Chin $J$ Radiol 2009; 34:191-96.

8. Suh J, Kennedy D. Treatment Options for Chronic Rhinosinusitis. Proc Am Thorac Soc 2011.8:132140
} 